\title{
High glucose enhances CD39 expression in vascular endothelial cells
}

\author{
Sudawadee Kongkhum, Mudtika Fungkrajai, Sompoch Prajan, Narisa Kengtrong Bordeerat, \\ Kanyanath Piumngam, Pilaiwan Siripurkpong \\ Faculty of Allied Health Sciences, Thammasat University, Pathumthani 12120, Thailand
}

\begin{abstract}
Background: Diabetes mellitus (DM) patients lose their ability to control normal blood glucose levels, resulting in high blood glucose levels (hyperglycemia). Hyperglycemia causes DM complications. This involves responses of vascular endothelial cells (VECs) to hyperglycemia, affecting inflammatory process and platelet activity. Ecto-enzyme CD39 is expressed on VECs, catalyzing the hydrolysis of ATP and ADP to AMP and, consequently, regulating inflammatory process and platelet activation.

Objective: We studied whether high glucose concentration has an effect on CD39 expression on VECs.

Methods: Cultured human umbilical vein endothelial cells (HUVEC) were used as a model of study. HUVEC were cultured in different glucose conditions $(4,9,24$, and $34 \mathrm{mM})$ for 24 hours. Cell viability was assessed using a 3-(4,5-dimethylthiazol-2-yl)-2,5-diphenyltetrazolium bromide (MTT)-based assay and expression of CD39 was examined by using SDS-PAGE and western blot techniques.

Results: HUVEC were cultured in normal (4 and $9 \mathrm{mM}$ ) or high (24 and $34 \mathrm{mM}$ ) glucose concentrations for short term (24 hours). The results showed that high glucose $(24$ and $34 \mathrm{mM})$ reduced cell viability to $89.5 \pm 11.3$ and $86.3 \pm 13.5$ (mean $\pm \mathrm{SD}$ ), compared with control $(4 \mathrm{mM})$, respectively. High glucose also induced increases in CD39 expression in HUVEC.

Conclusion: High glucose decreases cell viability and increases CD39 expression in HUVEC, suggesting involvement of CD39 in cell responses to high glucose.
\end{abstract}

Keywords: CD39, cell viability, diabetes mellitus, HUVEC, hyperglycemia, short term, vascular endothelial cell

Diabetes mellitus (DM) is a group of metabolic disorders of carbohydrate metabolism, causing health problems worldwide [1]. Human blood glucose levels are normally dynamic, increasing after meal and returning to normal after the action of insulin. However, blood glucose levels in DM patients are considerably higher than normal. The cause of DM is a deficiency of insulin hormone; therefore, DM patients are unable to maintain normal blood glucose levels, resulting in high blood glucose concentration (hyperglycemia). Chronic hyperglycemia can lead to microvascular and macrovascular complications, including diabetic retinopathy, nephropathy, neuropathy, and cardiovascular diseases [2,3]. A major player in underlying mechanisms involves vascular endothelial cells (VECs) [4, 5]. Hyperglycemia induces increases in expression of adhesion molecules on VECs, facilitating leukocyte infiltration and progress in

Correspondence to: Sudawadee Kongkhum, Faculty of Allied Health Sciences, Thammasat University, Pathum Thani 12120, Thailand.E-mail: sudakk@tu.ac.th pathogenesis of atherosclerosis. Moreover, activated VECs reduce their production of prostacyclin and NO, which are platelet-inhibitory substances. Hyperglycemia induces VECs responses, including oxidative stress, upregulation of adhesion molecules, and decreased production of prostacyclin and NO. Consequently, these effects lead to proinflammatory and prothrombotic states [6]. Because high glucose concentration induces oxidative stress and increased expression of adhesion molecules on VEC, we examined if high glucose concentrations have effects on VEC. First, we tested effects of high glucose concentrations on cell viability, using HUVEC as our model of VECs.

CD39 (EC 3.6.1.5, apyrase, ATPDase, Bgp95, G28-8, NTPDase-1 (nucleoside triphosphate diphosphohydrolase 1), vascular ATP diphosphohydrolase) is a member of the ecto-nucleoside triphosphate diphospohydolase (E-NTPDase) family, catalyzing hydrolysis of ATP, a proinflammatory mediator, and ADP, a platelet agonist, to AMP [7-9]. CD39 is expressed on several cells, including VECs, 
activated B cells, natural-killer cells, macrophages, dendritic cells, neurons, glial cells and astrocytes. CD39 has roles on inflammatory process and regulation of platelet activation [10-12]. Consequently, CD39 has been proposed as a potential target for treatment of patients with complications caused by platelet activation [11-13]. CD39 is an ecto-enzyme expressed on VECs and is an important player in the regulation of inflammation and platelet activation, associated with DM complications [14, 15]. However, it has not been reported if hyperglycemia has effects of CD39 in VECs. In the present study, we examined if high glucose can modulate CD39 expression in VECs.

\section{Materials and methods Cell culture}

Human umbilical vein endothelial cells (HUVEC) were used as a model of VECs. They were purchased from Invitrogen (Carlsbad, CA, USA) and cultured in M200 media supplemented with of $2 \%$ low serum growth supplement (LSGS) at $37^{\circ} \mathrm{C}$ in $5 \% \mathrm{CO}_{2}$. The media was changed every $48-72$ hours. At confluence, the media was changed to M200 media supplemented with 1\% LSGS. Random blood glucose levels are normally lower than $11.1 \mathrm{mM}$. Therefore, we chose 2 normal (4 and $9 \mathrm{mM})$ and 2 high $(24$ and $34 \mathrm{mM})$ concentrations of glucose and tested their effects. After treatment for 24 hours, cells were analyzed for cell viability. Glucose $(5,20$ and $30 \mathrm{mM})$ was then added to the media where the basal glucose concentration was $4 \mathrm{mM}$, giving final concentrations of $4,9,24$, and $34 \mathrm{mM}$. To test if hyperosmolarity in high glucose condition causes effects on VEC, osmolarity control was conducted by adding mannitol $(30 \mathrm{mM})$, representing the same osmolarity as $34 \mathrm{mM}$ glucose. Cells were then incubated at $37^{\circ} \mathrm{C}$ for 24 hours, and analyzed for cell viability and expression of CD39.

\section{Cell viability}

After treatment, cell viability was examined using a 3-(4,5-dimethylthiazol-2-yl)-2,5-diphenyltetrazolium bromide (MTT)-based assay [16, 17]. MTT solution ( $5 \mathrm{mg} / \mathrm{mL}$, Sigma-Aldrich, St. Louis, MO, USA) was added to cultured cells. After 4 hours incubation, MTT formazan was solubilized with DMSO and absorbance was determined at $570 \mathrm{~nm}$ and $630 \mathrm{~nm}$ as a reference wavelength. Percent cell viability was calculated by comparison with control by setting the mean absorbance of controls as $100 \%$.

\section{Expression of CD39}

Treated cells were harvested, lysed, and analyzed using SDS-PAGE and western blotting. Briefly, cell lysate was loaded onto a $10 \%$ SDS-polyacrylamide gel and separated proteins were then transferred to a nitrocellulose membrane. After that, the membrane was blocked with 5\% albumin-containing Tris-buffered saline (TBS), washed with TBS, incubated with primary antibody against CD39 (1:500, Santa Cruz, Santa Cruz, CA, USA) or $\beta$-actin (1:500, Santa Cruz), washed with TBS and finally incubated with anti-rabbit or anti-mouse $\operatorname{IgG}$ horse radish peroxidase (HRP) conjugates $(1: 10,000)$, respectively. The protein signals were then developed with peroxide and luminol enhancer solution (ECL SuperSignal Substrate kit, Pierce, Rockford, IL, USA) and visualized by exposure to X-ray films.

\section{Results}

We found that cell viability after treatment of glucose at 9,24 , and $34 \mathrm{mM}$ was $110.1 \pm 22.0 \%$, $89.5 \pm 11.3 \%$, and $86.3 \pm 13.5 \%$ (mean $\pm \mathrm{SD}$ ), respectively, compared with $4 \mathrm{mM}$ glucose (Figure 1). Moreover, cell viability of HUVEC treated with mannitol, as osmolarity control, was $94.7 \pm 15.7 \%$, which was not significantly different from normal glucose effects. A $t$ test showed the effects of 4 and $9 \mathrm{mM}$ glucose on cell viability were not significantly different, while effects of $24 \mathrm{mM}$ and $34 \mathrm{mM}$ glucose were significantly different from $9 \mathrm{mM}$ glucose $(p<0.05)$, but not different from $4 \mathrm{mM}$ glucose ( $p=0.09$ and 0.05 , respectively).

We observed that short-term exposure of high glucose $(34 \mathrm{mM})$ caused increases in expression of CD 39 in HUVEC, while mannitol, an osmolarity control, did not induced changes (Figure 2).

\section{Discussion}

We found that cell viability reduced significantly by high glucose concentration ( 24 and $34 \mathrm{mM}$ ). This finding is similar to that of previous studies, showing that high glucose concentrations decrease VEC viability and increase apoptosis $[18,19]$. However, the earlier studies used long-term exposure to high glucose concentrations. In the present study, we used shortterm exposure (24 hours) and observed similar results. This reveals that short-term exposure to hyperglycemia can also cause adverse effects to VECs. Previous studies also reported effects of shortterm exposure to hyperglycemia [20-22]. High glucose induces oxidative stress in endothelial cells after 3- 


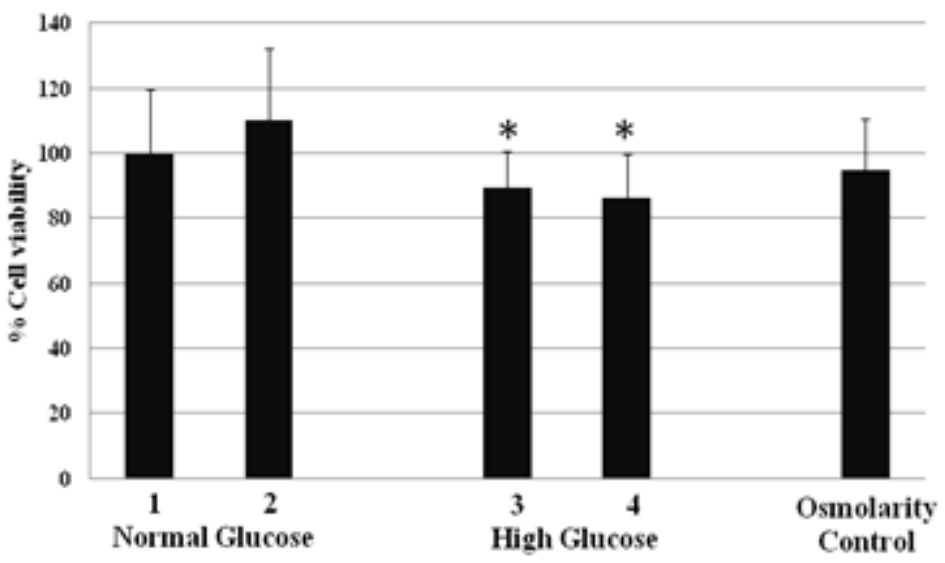

Figure 1. Effects of high glucose on cell viability. HUVECs were cultured in different glucose concentrations for 24 hours; 4 (1) and 9 (2) $\mathrm{mM}$ as normal glucose levels and 24 (3) and 34 (4) mM as high glucose levels. Osmolarity control was achieved using mannitol at the same concentration as $34 \mathrm{mM}$ glucose. Cell viability was determined by the MTT method. The data are shown as percent, compared with basal medium glucose at $4 \mathrm{mM}(100 \%)$. ${ }^{*}$ Cell viability significantly different from normal glucose $(p<0.05)$. The data are from three triplicate experiments.

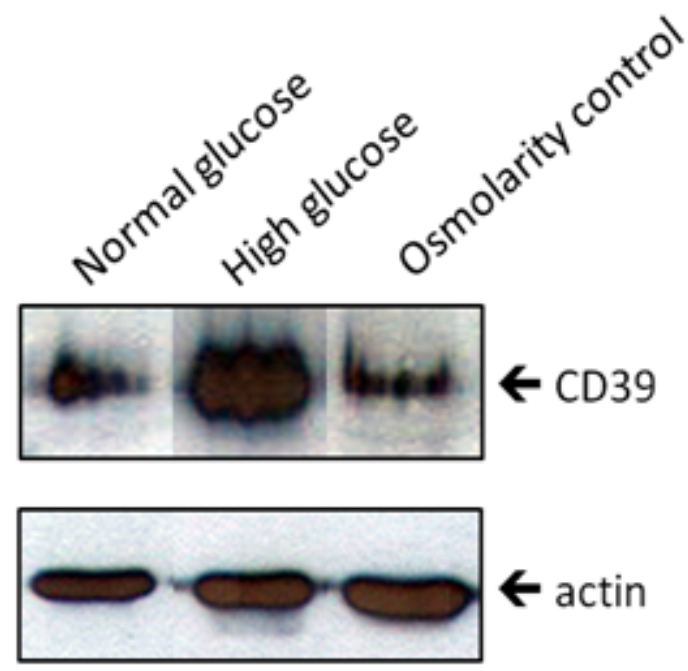

Figure 2. Effects of high glucose on CD39 expression. HUVECs were cultured in normal (4 mM), high (34 mM) glucose concentrations or mannitol (osmolarity control) for 24 hours. CD39 expression was assessed by the SDSPAGE and western blotting. The upper panel shows CD39 bands and the lower shows bands from $\beta$-actin loading controls. The data represent 6 different experiments.

hour exposure and enhances expression of adhesion molecules (E-LAM-1, VCAM-1 and ICAM-1) after 24-hour exposure [20, 22]. In addition, an in vivo study in C57B1/6J mice showed that acute hyperglycemia results in increases in oxidative stress and worsened myocardial infarction [21]. Moreover, another in vivo study in DM patients revealed that induction of oxidative stress is associated with acute glucose fluctuation, indicated by using the mean amplitude of glycemic excursion (MAGE, arithmetic mean of the difference between peak and nadir glucose levels). Acute glucose fluctuation reflects exposure to high glucose, at least, for a short period of time [23]. They also showed that the oxidative stress is not associated with long-term exposure of high glucose $\left(\mathrm{HbA}_{1 \mathrm{C}}\right)$. This suggests that short-term exposure to high glucose concentrations, including controlled DM patients, can have impact on the patients. These reports support our finding that short-term exposure to high glucose can reduce HUVEC viability. 
We found that a high glucose concentration induced an increase in CD39 expression after shortterm exposure (24 hours) to high glucose $(34 \mathrm{mM})$. Moreover, hyperosmolarity (osmolarity control) did not cause a reduction of cell viability or change of CD39 expression; therefore, these effects appear to be associated with high glucose, not increased osmolarity.

In the present study, high glucose caused several fold increases in CD39 expression, compared with that of controls, while high glucose induces about $10 \%-20 \%$ decreases in cell viability (cell death). The later result is consistent with previous reports. Because the changes of responses in cell viability and CD39 expression are in different magnitude, we consider that cell viability or cell death is not a causes of change in CD39 expression. However, further study is required to understand how high glucose induced increases in CD39. It is possible that augmentation of CD39 is a mechanism to protect vascular endothelial cells from thrombosis as a result of inflammation and platelet activation, because CD39 helps hydrolyze the inflammatory mediator ATP and platelet agonist ADP. CD39 helps prevent diabetic nephropathy by suppressing upregulation of adhesion molecules on endothelial cells, resulting in reduction of leukocyte infiltration, suppression of scavenger receptors, limitation of foam cell formation, a step in atherogenesis, and prevention of platelet activation [10]. This concept is supported by studies showing that CD39 is upregulated by several insults to endothelial cells, including lipopolysaccharide and proinflammatory cytokines [24, 25]. In addition, it has been shown that increases in CD39 on glial cells of retina in diabetic mice [26], and on platelets and peripheral blood mononuclear cells (PBMC), isolated from diabetic patients $[27,28]$.

\section{Conclusion}

CD39 with a role in endothelial cell responses to insults, is modulated by high glucose conditions, suggesting its involvement in cell responses to high glucose or hyperglycemia. Short-term exposure to high glucose mediates effects on HUVEC and suggest that CD39 is involved in HUVEC responses to the exposure to hyperglycemia.

\section{Acknowledgements}

This work was financially supported by Thammasat University. None of the authors declared any conflict of interest.

\section{References}

1. Chen L, Magliano DJ, Zimmet PZ. The worldwide epidemiology of type 2 diabetes mellitus-present and future perspectives. Nat Rev Endocrinol. 2012; 8: 228-36.

2. Brownlee M. Biochemistry and molecular cell biology of diabetic complications. Nature. 2001; 414:813-20.

3. Giacco F, Brownlee M. Oxidative stress and diabetic complications. Circ Res. 2010; 107:1058-70.

4. Rask-Madsen C, King GL. Vascular complications of diabetes: mechanisms of injury and protective factors. Cell Metab. 2013; 17:20-33.

5. van den Oever IA, Raterman HG, Nurmohamed MT, Simsek S. Endothelial dysfunction, inflammation, and apoptosis in diabetes mellitus. Mediators Inflamm. 2010; 2010:792393.

6. Schafer A, Bauersachs J. Endothelial dysfunction, impaired endogenous platelet inhibition and platelet activation in diabetes and atherosclerosis. Curr Vasc Pharmacol. 2008; 6:52-60.

7. Robson SC, S vigny J,Zimmermann H. The E-NTPDase family of ectonucleotidases: Structure function relationships and pathophysiological significance. Purinergic Signal. 2006; 2:409-30.

8. Wang TF, Guidotti G. CD39 is an ecto- $\left(\mathrm{Ca}^{2+}, \mathrm{Mg}^{2+}\right)-$ apyrase. J Biol Chem. 1996; 271:9898-901.

9. Wang TF, Guidotti G. Widespread expression of ectoapyrase (CD39) in the central nervous system. Brain Res. 1998; 790:318-22.

10. Dwyer KM, Deaglio S, Gao W, Friedman D, Strom TB, Robson SC. CD39 and control of cellular immune responses. Purinergic Signal. 2007; 3:171-80.

11. Atkinson B, Dwyer K, Enjyoji K, Robson SC. Ectonucleotidases of the CD39/NTPDase family modulate platelet activation and thrombus formation: Potential as therapeutic targets. Blood Cells Mol Dis. 2006; 36 : 217-22.

12. Marcus AJ, Broekman MJ, Drosopoulos JH, Islam N, Alyonycheva TN, Safier LB, et al. The endothelial cell ecto-ADPase responsible for inhibition of platelet function is CD39. J Clin Invest. 1997; 99:1351-60.

13. Koziak K, Sevigny J, Robson SC, Siegel JB, Kaczmarek E. Analysis of CD39/ATP diphosphohydrolase (ATPDase) expression in endothelial cells, platelets and leukocytes. Thromb Haemost. 1999; 82:1538-44.

14. Michno A, Bielarczyk H, Pawelczyk T, JankowskaKulawy A, Klimaszewska J, Szutowicz A. Alterations of adenine nucleotide metabolism and function of blood platelets in patients with diabetes. Diabetes. 2007; 56:462-7. 
15. Sobol AB, Watala C. The role of platelets in diabetesrelated vascular complications. Diabetes Res Clin Pract. 2000; 50:1-16.

16. Mosmann T. Rapid colorimetric assay for cellular growth and survival: application to proliferation and cytotoxicity assays. J Immunol Methods. 1983; 65: 55-63.

17. Oez S, Platzer E, Welte K. A quantitative colorimetric method to evaluate the functional state of human polymorphonuclear leukocytes. Blut. 1990; 60:97-102.

18. Ido Y, Carling D, Ruderman N. Hyperglycemia-induced apoptosis in human umbilical vein endothelial cells: inhibition by the AMP-activated protein kinase activation. Diabetes. 2002; 51:159-67.

19. Leal EC, Aveleira CA, Castilho AF, Serra AM, Baptista FI, Hosoya K, et al. High glucose and oxidative/nitrosative stress conditions induce apoptosis in retinal endothelial cells by a caspaseindependent pathway. Exp Eye Res. 2009; 88:983-91.

20. Altannavch TS, Roubalova K, Kucera P, Andel M. Effect of high glucose concentrations on expression of ELAM-1, VCAM-1 and ICAM-1 in HUVEC with and without cytokine activation. Physiol Res. 2004; 53:77-82.

21. Yang Z, Laubach VE, French BA, Kron IL. Acute hyperglycemia enhances oxidative stress and exacerbates myocardial infarction by activating nicotinamide adenine dinucleotide phosphate oxidase during reperfusion. J Thorac Cardiovasc Surg. 2009; 137:723-9.

22. Yano M, Hasegawa G, Ishii M, Yamasaki M, Fukui M, Nakamura N, et al. Short-term exposure of high glucose concentration induces generation of reactive oxygen species in endothelial cells: implication for the oxidative stress associated with postprandial hyperglycemia. Redox Rep. 2004; 9:111-6.

23. Monnier L, Mas E, Ginet C, Michel F, Villon L, Cristol $\mathrm{JP}$, et al. Activation of oxidative stress by acute glucose fluctuations compared with sustained chronic hyperglycemia in patients with type 2 diabetes. JAMA. 2006;295:1681-7.

24. Favaloro EJ. Differential expression of surface antigens on activated endothelium. Immunol Cell Biol. 1993; 71:571-81.

25. Kapojos JJ, van den Berg A, Borghuis T, Banas B, Huitema S, Poelstra K, et al. Enhanced ecto-apyrase activity of stimulated endothelial or mesangial cells is downregulated by glucocorticoids in vitro. Eur $\mathrm{J}$ Pharmacol. 2004; 501:191-8.

26. Wurm A, Iandiev I, Hollborn M, Wiedemann P, Reichenbach A, Zimmermann H, et al. Purinergic receptor activation inhibits osmotic glial cell swelling in the diabetic rat retina. Exp Eye Res. 2008; 87:385-93.

27. Lunkes GI, Lunkes DS, Leal D, Araujo Mdo C, Correa $\mathrm{M}$, Becker L, et al. Effect of high glucose levels in human platelet NTPDase and 5'-nucleotidase activities. Diabetes Res Clin Pract. 2008; 81:351-7.

28. Garcia-Hernandez MH, Portales-Cervantes L, CortezEspinosa N, Vargas-Morales JM, Fritche Salazar JF, Rivera-Lopez E, et al. Expression and function of P2X(7) receptor and CD39/Entpd1 in patients with type 2 diabetes and their association with biochemical parameters. Cell Immunol. 2011; 269:135-43. 